\title{
Synergistic Smart Morphing Aileron: Aero-structural Performance Analysis
}

\author{
Alexander M Pankonien ${ }^{1}$, Karthik Duraisamy ${ }^{2}$, Cassio T. Faria ${ }^{3}$, and Daniel J. Inman ${ }^{4}$ \\ Department of Aerospace Engineering, University of Michigan, Ann Arbor, Michigan, 41809, USA
}

\begin{abstract}
Combining multiple smart materials into a single concept that effectively utilizes their unique capabilities can achieve performance beyond the individual limitations of its constituent materials. Previous work characterized the mechanisms of the Synergistic Smart Morphing Aileron (SSMA) concept, which combines the fast, conformal actuation of MacroFiber Composites (MFC) with the high specific work of Shape Memory Alloys (SMA). The aerodynamic benefits of the SSMA concept are identified by comparing the performance of the combined system with its constituent actuators for a representative airfoil. The optimized uncoupled performance of experimentally measured shapes in a 2D Navier-Stokes simulation showed $45 \%$ improvement in ability to affect the flow over a range of flight conditions. A geometrically nonlinear finite element code was then coupled with the flow simulation confirming improved performance under aerodynamic loads. Additionally, the SSMA demonstrated the ability to mitigate aeroelastic effects and flow separation near stall via novel reflex actuation. These results show a combined system with improved flow control beyond its constituent subsystems.
\end{abstract}

\section{Nomenclature}

$\begin{array}{ll}\alpha & =\text { angle of attack } \\ c & =\text { chord of airfoil } \\ C_{d} & =\text { two-dimensional drag coefficient } \\ C_{l} & =\text { two-dimensional lift coefficient } \\ C_{m} & =\text { two-dimensional pitching moment coefficient } \\ C_{p} & =\text { two-dimensional pressure coefficient } \\ M F C_{\delta} & =\text { normalized Macro-Fiber Composite actuation } \\ S M A_{\delta} & =\text { normalized Shape Memory Alloy actuation } \\ V_{\infty} & =\text { freestream flow speed }\end{array}$

\section{Introduction}

$\mathrm{T}$ RADITIONAL solutions to aerodynamic shape change involve articulated surfaces that use multiple hinges, actuators, structural mechanisms and sliding surfaces. The resultant structure may improve aerodynamic performance, but often at the expense of significantly increased complexity and weight as well as significant energy cost. Smart material morphing concepts seek to improve aerodynamic performance without these penalties via a novel method: acting as both structure and actuator. By combining the loaded structure, aerodynamic surface and actuation mechanism, prohibitive additions to weight and complexity are avoided while still increasing the overall aerodynamic performance ${ }^{1}$. However, by integrating the actuation device into the structure, the morphing concept becomes constrained by the inherent material limitations of the smart material. For example, shape memory alloys (SMA) typically have limited frequency response, but large actuation strain and high blocking stress, as seen in Figure 1. However, piezoelectric materials (shown as PZT) have high bandwidth but low actuation strain.

\footnotetext{
${ }^{1}$ PhD Candidate, Department of Aerospace Engineering, 1320 Beal Avenue, AIAA Student Member.

${ }^{2}$ Assistant Professor, Department of Aerospace Engineering, 1320 Beal Avenue, AIAA Member.

${ }^{3}$ Post-Doctoral Scholar, Department of Aerospace Engineering, 1320 Beal Avenue, AIAA Member.

${ }^{4}$ Collegiate Professor and Chair, Department of Aerospace Engineering, 1320 Beal Avenue, AIAA Fellow.
} 

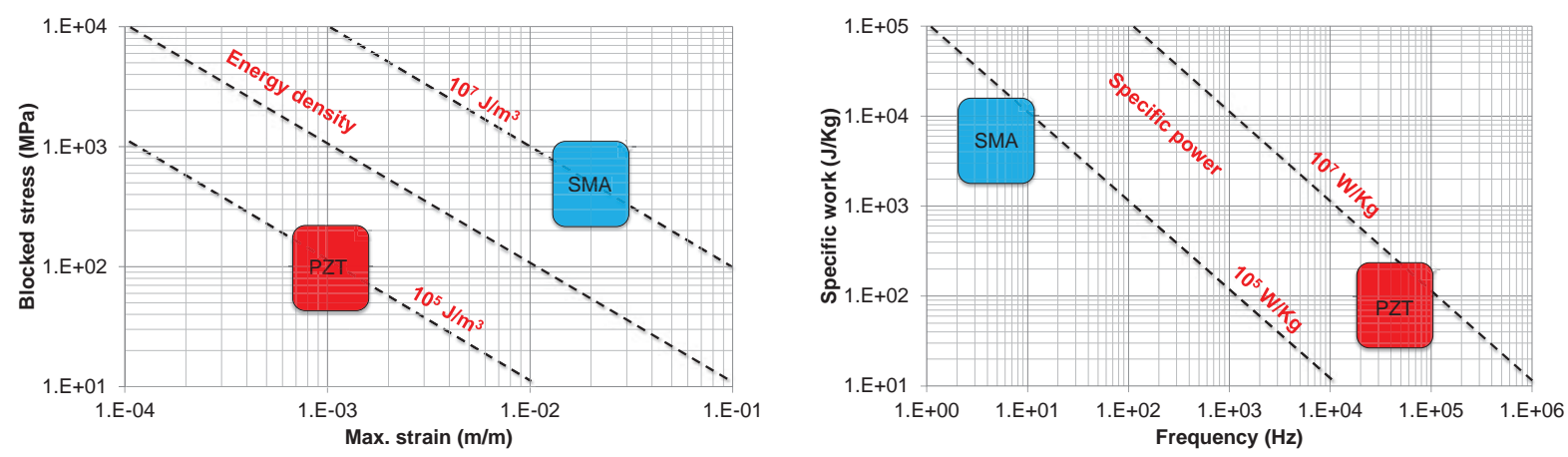

Figure 1: Comparison of smart material actuators (Adapted from - NASA/JPL/EAP website)

Previous actuation concepts have attempted to overcome these limitations by combining smart materials in a single design to compensate for each other's limitations and leveraging a mismatch in material actuation attributes. Chinaud et. al used piezostacks combined with SMA wires to actuate rigid flaps to control vortices of different timescales in a flow over a plate ${ }^{2}$. Similarly, Hines et. al actively controlled the stiffness of a morphing structure using shape memory polymers while a piezoelectric bimorph actuated a flapping mechanism ${ }^{3}$. Despite using predeveloped actuators to prevent the design process from becoming overly complex, the number of such applications has been limited.

Similarly to the previous concepts, the Synergistic Smart Morphing Aileron (SSMA) concept ${ }^{4}$ combined a SMAdriven hinge and piezoelectrically-driven Flexure Box aileron into a single morphing application, leveraging the difference in actuation mechanisms. The Flexure Box concept uses voltage-driven MFC unimorphs to create smooth, conformal morphing on a fast timescale ${ }^{5,6}$. Meanwhile, the SMA wires utilize a solid-state phase change to rotate the aileron on a slower timescale with greater resistance to aerodynamic loading and lower holding power.

\section{A. Scope of investigation and description of SSMA}

The goal of this paper is to identify how the SSMA morphing concept improves aerodynamic performance beyond that of its constituent actuators. These improvements are identified by examining the relative performance of the SSMA concept and its constituent actuators in a representative configuration.

For the representative SSMA implementation, shown in Figure 2, a NACA 0012 airfoil with a $305 \mathrm{~mm}$ chord was chosen. In this configuration, the aileron is rotated about a discrete hinge at $160 \mathrm{~mm}$ chord by two antagonistic pre-strained SMA wires (shown in blue), which are heated by a custom-built switching circuit. A rotary potentiometer (not depicted) is located at the discrete hinge to allow for positional feedback of the actuator. The Flexure Box ${ }^{7,4}$, developed from the hinged box concept ${ }^{8}$, is constructed via MFC unimorphs (shown in red) bonded to an elastomeric 3d-printed box, completing a solid state flexure mechanism that is capable of high precision positioning free of frictional losses. A piezoresistive flex sensor (not depicted) is embedded to allow for independent closed-loop positioning control of the Flexure Box. The MFCs are driven with an AVID Dual Channel MFC Bimorph High Voltage Driver. The outer airfoil surface is formed by adhering pre-strained silicone rubber (not depicted) via two-sided tape to the rigid leading edge (shown in black) and Flexure box.

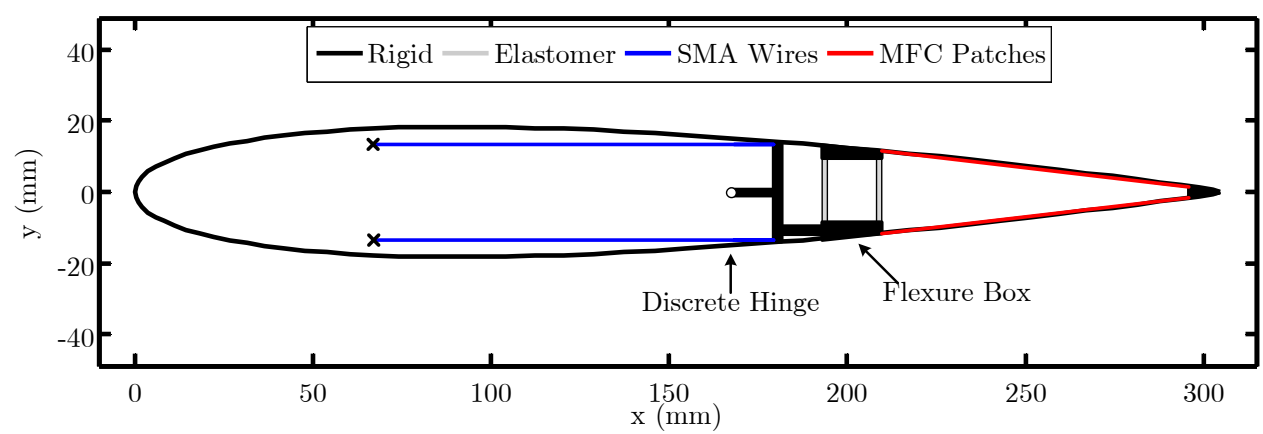

Figure 2: Diagram of Synergistic Smart Morphing Aileron Concept

American Institute of Aeronautics and Astronautics 


\section{Static, Uncoupled Aerodynamic Optimization}

The aerodynamic benefits of the SSMA concept are first investigated by modeling and optimizing the actuation's effect on the flow and comparing its performance with that of its constituent actuators.

\section{A. Uncoupled morphing simulation method}

To identify relevant aerodynamic shapes for modeling the Flexure Box, the achievable shapes were experimentally measured by cycling the box through its entire actuation range, free of aerodynamic loads. This was chosen to represent a bound as the best possible scenario where the structure would not be affected by aerodynamic loads. The maximum and minimum potential differences applied to the MFCs by the high voltage amplifier were $+1.5 \mathrm{kV}$ and $-0.5 \mathrm{kV}$, respectively. The amplifier is built to scale the voltage ratio $3: 1$ so that the negative voltage applied to actuate the unimorph in compression is proportional to the positive voltage applied to actuate the other unimorph in tension. Marker points were tracked over 41 steps (21 increasing, decreasing) in the actuation cycle using digital image correlation software. A representative image from one of the steps is shown in Figure 3.

The morphing trailing edge was then combined with a NACA 0012 leading edge profile to create a range of morphing airfoils. The y-coordinates for each of the airfoils were then plotted against airfoil arc length and smoothed to eliminate position measurement errors. An example of smoothed data and the resultant airfoil shape are shown in Figure 4.

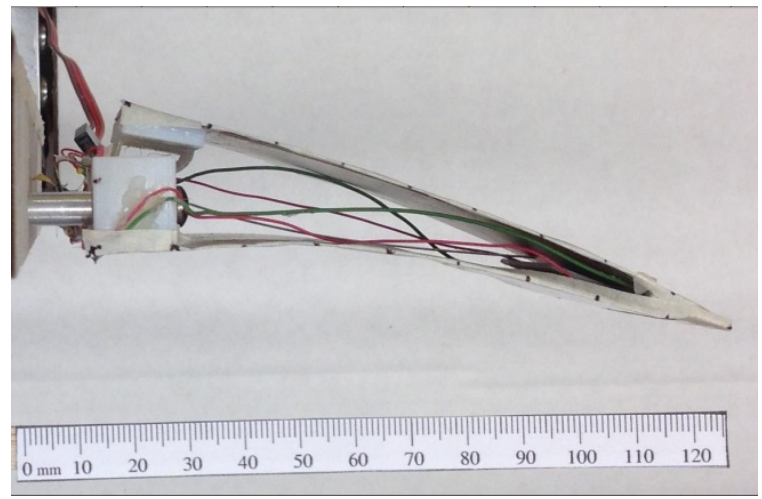

Figure 3: Representative Actuation

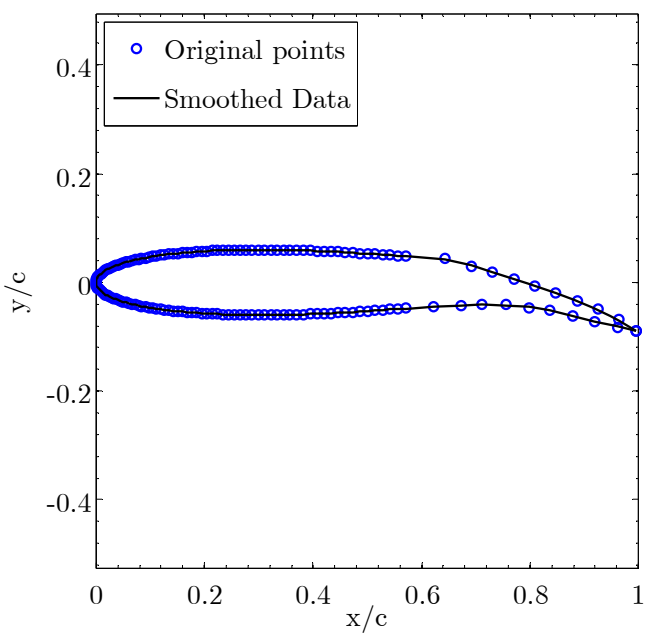

Figure 4: Resultant airfoil Shape

The MFCs are voltage controlled and for large actuation amounts experience hysteresis as seen in Figure 5, tracking the y-coordinate of the tip displacement with respect to input voltage. To evaluate the effect of the variation of these shapes on relevant aerodynamic quantities, the aerodynamics were simulated for these shapes using the compressible Unsteady Reynolds Averaged Navier-Stokes (URANS) equations.

The OVERTURNS code, which operates on structured overset meshes, was employed for this purpose. Figure 6 shows a sample mesh system for a representative simulation ${ }^{9,10}$. The numerical method uses a third order accurate upwind scheme to compute the inviscid fluxes, while the viscous terms are discretized using second order accurate central differencing. Low Mach number pre-conditioning ${ }^{11}$ is used to accelerate convergence within the context of implicit time integration. For turbulence closure, the Spalart-Allmaras ${ }^{12}$ turbulence model is employed. This formulation has been validated extensively over a wide range of flow conditions and geometries ${ }^{13}$ including in the transitional and turbulent range of Reynolds numbers ${ }^{14}$. 


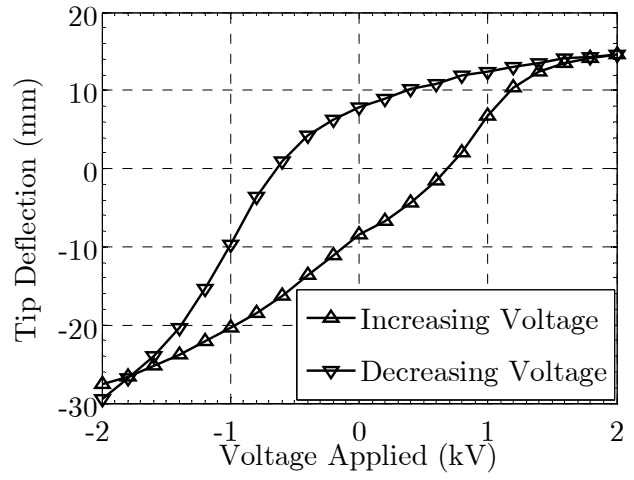

Figure 5: Hysteresis of tip deflection

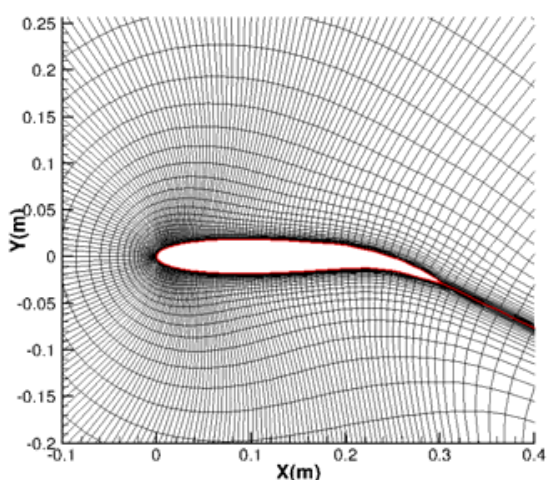

Figure 6: Close-up of fluid mesh

The aerodynamic performance of all 41 experimentally measured shapes were evaluated at 3 different relevant flow speeds $(10,20,30 \mathrm{~m} / \mathrm{s})$ with 3 different angles of attack $\left(-15^{\circ}, 0^{\circ}, 15^{\circ}\right)$. The mean relative error accumulated by ignoring the effects of actuation hysteresis on lift, drag, and lift to drag ratio was linearly interpolated and plotted in Figure 7. Using this data, it was assumed that the tip deflection alone would be an adequate indicator of actuation state of the Flexure Box concept and that the error incurred by this assumption would be small enough to be disregarded for initial performance estimates.
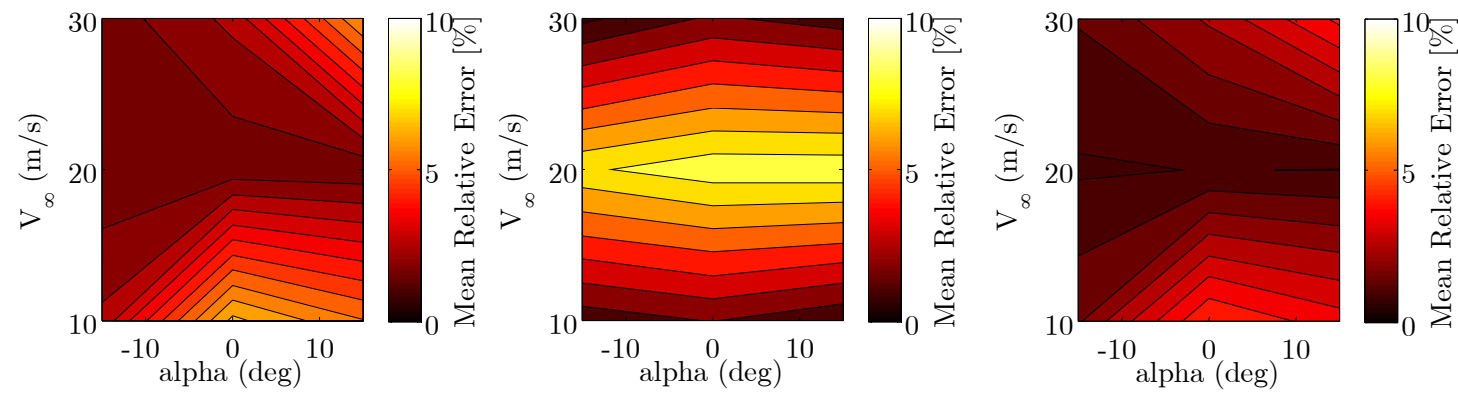

Figure 7: Relative error in experimental data due for (left) $C_{L}$ (middle) $C_{D}$ (right) $C_{M}$

\section{B. Parametric description of the SSMA airfoil}

The actuation of the Flexure Box concept, through previous assumptions, was thus described exclusively by the tip deflection, simplifying the actuation space. Accordingly, only half of the flexure box experimental data shapes were necessary to describe all possible morphing shapes. A normalized actuation, $\mathrm{MFC}_{\delta}$, was created which rescaled the tip deflection to the range [-1,1], which maps to full actuation tip down and full actuation tip up, respectively.

The tip deflection induced by the SMA-driven hinge (measured previously experimentally ${ }^{4}$ ) was mapped to a normalized actuation, $\mathrm{SMA}_{\delta}$, in a similar manner. Although the SMA wires require pre-stress when built, it was assumed that the range for the SMA-driven hinge is centered on 0 degrees. The mapping of normalized actuation for $\mathrm{MFC}_{\delta}$ and $\mathrm{SMA}_{\delta}$ is then as follows in Figure 8. Hereafter, the actuation mechanisms are denoted by their smart material actuator, when needed for brevity. Note that the relationships between the tip deflection that are depicted are true for the SSMA concept only for the case where the other actuation mechanism is unactuated. The two different actuation mechanisms can be combined such that the SMA and MFC actuate together or in opposite directions to create an effective reflex camber. The actuators have no effect on the airfoil geometry when the tip deflection for both individual concepts are zero, which can be found in the previous plot as $\left[\mathrm{MFC}_{\delta}, \mathrm{SMA}_{\delta}\right]=$ $[0.3267,0]$. The MFC has a non-zero center for its normalized actuation due to the linear mapping and the asymmetry of the MFC's range. The range of possible shapes over the total space of the SSMA airfoil can then be seen by viewing the bounds of the actuation domain, seen in Figure 9. 


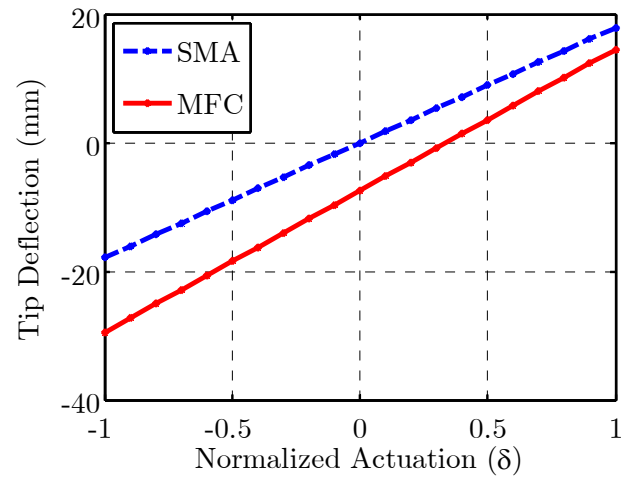

Figure 8: Correlation between tip deflection and normalized actuation
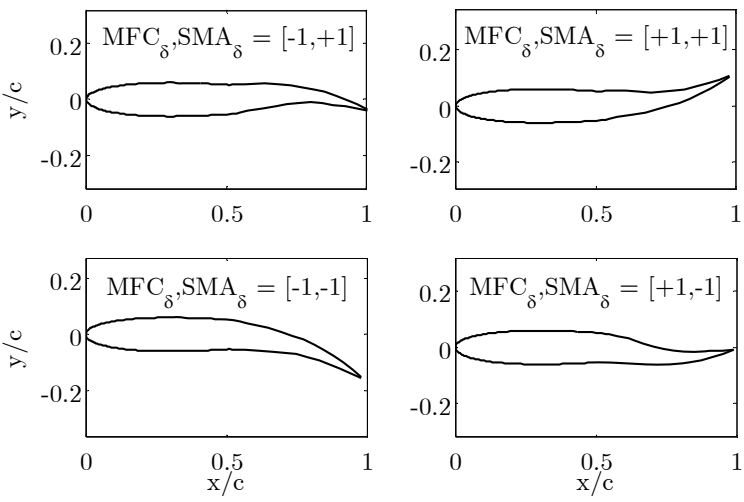

Figure 9: Bounds of SSMA actuation domain

\section{Effect of SSMA actuation on aerodynamic parameters}

The parametric SSMA description was used to create a sampling of 441 different airfoil shapes, spaced evenly as a grid in the normalized actuation domain. A representative flow speed $\left(\mathrm{V}_{\infty}=20 \mathrm{~m} / \mathrm{s}\right)$ and angle of attack $\left(\alpha=15^{\circ}\right)$ were chosen to illustrate the complex effect of the actuation domain on aerodynamic forces. The results from the steady Navier-Stokes simulations are shown below in Figure 10.
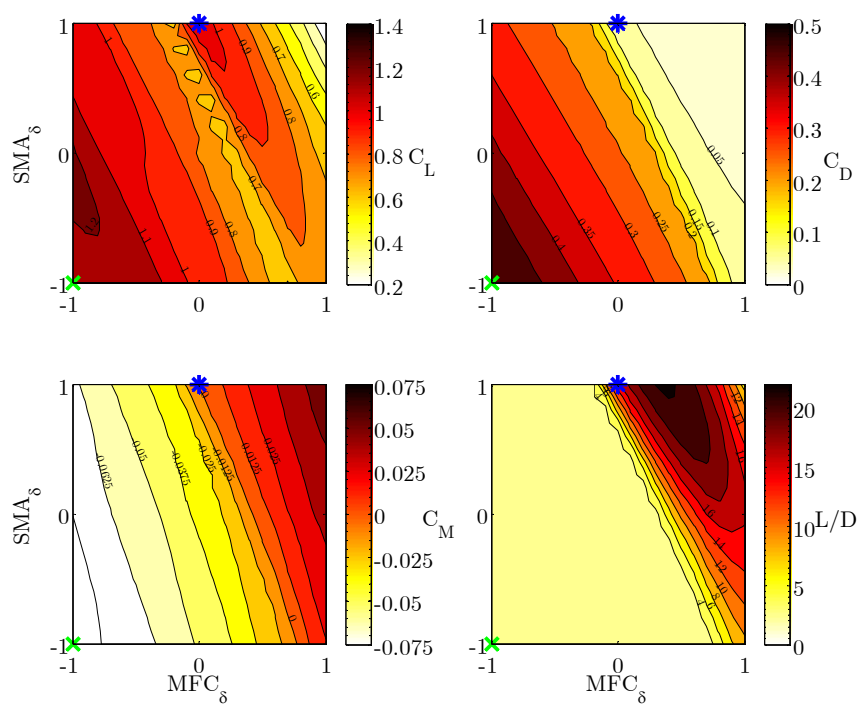

Figure 10: Aerodynamic forces over actuation range for $V_{\infty}=20 \mathrm{~m} / \mathrm{s}, \alpha=\mathbf{1 5}^{\circ}$

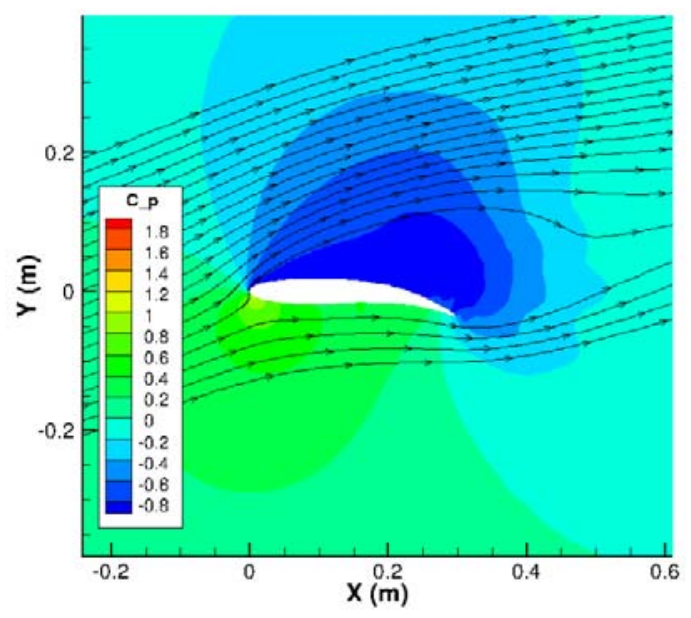

Figure 11: Field of pressure coefficient for $\left[\mathrm{MFC}_{\delta}, \mathrm{SMA}_{\delta}\right]=[-1,-1]$.

Near stall, the lift coefficient is shown to have a constrained, local maximum at $\left[\mathrm{MFC}_{\delta}, \mathrm{SMA}_{\delta}\right]=[0,1]$, noted in Figure 10 as a blue "*" and an global maximum at $\left[\mathrm{MFC}_{\delta}, \mathrm{SMA}_{\delta}\right]=[-1,-1]$, noted as a green " $\mathrm{x}$ ". The constrained maximum is actually a case of reflex actuation, because from the previous mapping, the unactuated configuration is $\left[\mathrm{MFC}_{\delta}, \mathrm{SMA}_{\delta}\right]=[0.3267,0]$. Actuating further downward causes flow separation seen by a sharp line in all four contours. The flow separation increases lift after an initial decrease, dramatically increases the drag coefficient, decreases the lift to drag ratio, and increases the pitching moment. To visualize the flow separation, the pressure field and streamlines for the global maximum lift condition are shown in Figure 11. As a result, when determining optimal actuator configuration, it will be shown that it is important to take into account these effects caused by flow separation. 


\section{Uncoupled aerodynamic optimization results}

A range of flight conditions (angles of attack) were tested to compare the static aerodynamic performance of the SSMA concept to that of its constituent elements. A simple optimizer was used to improve the speed at which the optimal performance was found for each flight condition. A gradient-free Nelder-Mead Simplex optimizer ${ }^{15}$ was employed with a merit function that penalized actuation points outside of the domain. A gradient free method was chosen due to the discontinuities in aerodynamic parameters seen previously and the black-box nature of the compiled fluids code and the limited number of designs variables. The aerodynamic performance was optimized to find the maximum and minimum lift coefficient over a range of angles of attack and a single representative flow speed $(20 \mathrm{~m} / \mathrm{s})$. The lift coefficient was chosen as a metric of the actuators to influence the flow because of the application of airfoils to providing lift in flight and the complexity of the effect of flow separation on performance. A summary of the optimizations follows.

$$
\begin{array}{ll}
\text { Maximize, Minimize: } & f\left(M F C_{\delta}, S M A_{\delta}\right)=C_{l} \\
\text { Subject to the constraints: } & -1 \leq M F C_{\delta} \leq 1 \\
& -1 \leq S M A_{\delta} \leq 1 \\
\text { Given: } & \mathrm{c}, \alpha, \mathrm{V}_{\infty}
\end{array}
$$

Three different cases were chosen to simulate the combined system and its actuators: the SSMA system, the MFC only, and the SMA only. In the case of the MFC-only and SMA-only actuation, a single parameter goldensection optimizer was used with the actuation bounds as the initial search interval and the other actuator left unactuated. The results from the optimization are shown in Figures 12 and 13.

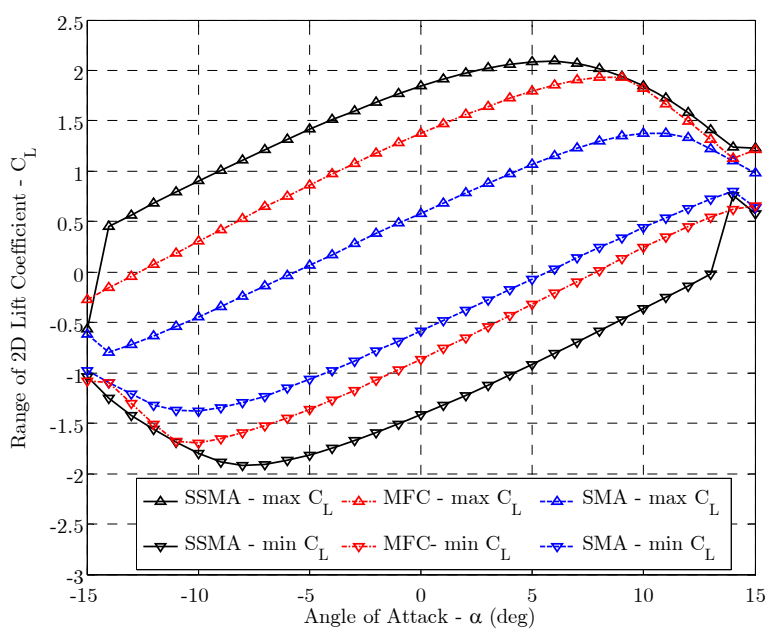

Figure 12: Range in achievable lift coefficients for SSMA and constituent subsystems

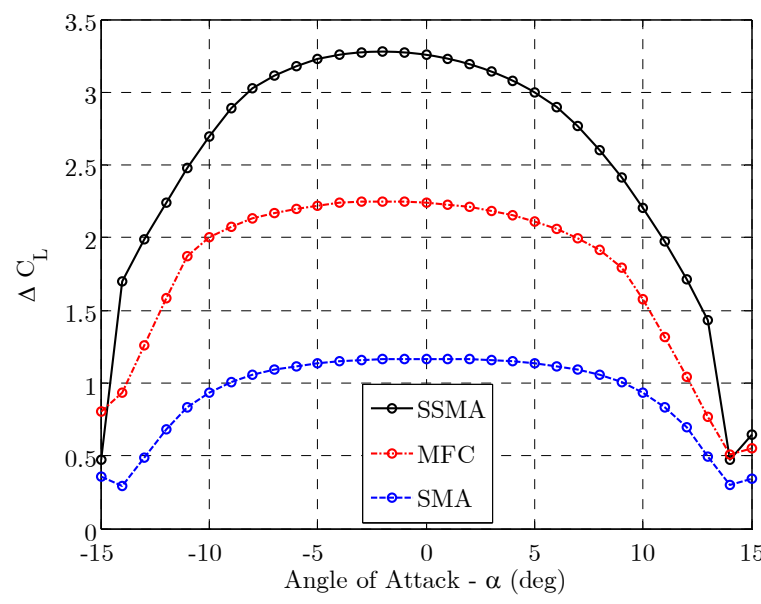

Figure 13: Achievable change in lift coefficients for SSMA and constituent subsystems

From the plots above, the SMA concept yields the smallest amount of lift coefficient range for a given angle of attack, which is symmetric about the vertical axis at $\alpha=0$ degrees, due to the symmetric constraint on deflection angle. The conformal morphing of the MFC yields a larger range than the SMA, albeit asymmetric due to the asymmetry of the flexure box actuation domain. Finally, the SSMA concept shows a much larger range, nearly equivalent to the addition of the two actuators. The improvement in maximum achievable range of lift coefficient over all flight conditions is show in the following table.

Table 1: Summary of Uncoupled Optimized Results

$\begin{array}{ccccccc} & \begin{array}{c}\text { Maximum } \\ \mathbf{C}_{\mathbf{L}}\end{array} & \begin{array}{c}\text { \% Improved } \\ \text { by SSMA }\end{array} & \begin{array}{c}\text { Minimum } \\ \mathbf{C}_{\mathbf{L}}\end{array} & \begin{array}{c}\text { \% Improved } \\ \text { by SSMA }\end{array} & \begin{array}{c}\text { Maximum } \\ \mathbf{\Delta \mathbf { C } _ { \mathbf { L } }}\end{array} & \begin{array}{c}\text { \% Improved } \\ \text { by SSMA }\end{array} \\ \text { SMA } & 1.378 & 51.7 \% & -1.378 & 39.5 \% & 1.168 & 180 \% \\ \text { MFC } & 1.932 & 8.2 \% & -1.696 & 13.4 \% & 2.250 & 45.6 \% \\ \text { SSMA } & 2.093 & - & -1.922 & - & 3.276 & -\end{array}$


The SSMA concept represents roughly a $50 \%$ improvement in maximum or minimum lift coefficient over the SMA hinge concept and roughly a $10 \%$ improvement over the MFC conformal morphing concept. However, these values only represent the absolute maximum and minimum. The results show that the SSMA concept roughly adds the abilities of the two actuation concepts to affect the flow, especially at off-maximum performance conditions with some losses $(\sim 10 \%)$ due to flow separation. The overall ability of the SSMA to affect the flow is improved $180 \%$ over the SMA and $50 \%$ over the MFC actuators alone.

\section{Static, Coupled Aerodynamic and Structural Optimization}

The uncoupled simulations showed that the MFC could affect flow nearly 100\% more effectively than the SMA. However, from previous experimental results noted that the compliance of the Flexure Box concept and MFC unimorphs experienced significant aeroelastic effects. Namely, the Flexure Box experienced reduction in tip deflection range up to $50 \%$ for flow speeds as low as 20 meters / second ${ }^{7}$.

To incorporate these deformations into the simulations, a structural finite element code was chosen to couple with the aerodynamic simulations. Modeling the aeroelastic effects on the structure was chosen primarily because it enabled the capability to dissect the complex structural and aerodynamic problem without affecting the test itself. Additionally, modeling eliminated the need for closed-loop control, necessary in implementing precise position control for real hysteretic actuators. A description of the development of the simulations follows.

\section{A. Implementation of corotational finite element code}

As part of the well-known Euler-Lagrangian coupling problem between CFD and FEM simulations, the structure is described as in the original configuration, but the aerodynamics loads act on the structure in the deformed configuration. Because of the relatively large actuator deformations and the relatively high fidelity of the NavierStokes simulations it was determined that the structure could not simply be represented as a static shape where the loaded and unloaded configurations are the same. Thus a non-linear structural code was needed to accurately model the aeroelastic effects.

A simple co-rotational finite element code was developed and used to keep the coupled problem from becoming prohibitively complex. The co-rotational framework is advantageous in that it separates the geometric nonlinearities associated with large displacements and rotations from the material model still allowing for the simplified material model of infinitesimal strain ${ }^{16}$. Previous work has shown that such a method can be quickly used to describe both static and dynamic aeroelastic effects on both rigid and flexible airfoils ${ }^{17}$.Using the corotational finite elements described by Crisfield ${ }^{18}$ and Battini ${ }^{16}$, a 2D cross-section of the SSMA airfoil using Euler-Bernoulli beam elements. The resultant model is shown in Figure 14.

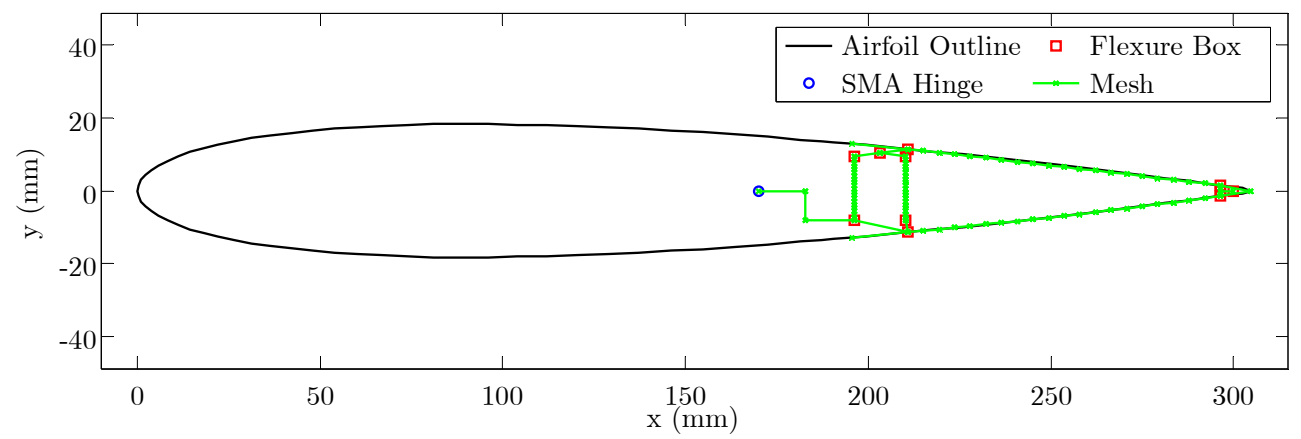

Figure 14: Overview of Finite Element Structure

For simplicity, the skin spanning the gap between the Flexure Box and the rigid leading edge of the airfoil was modeled as a wiper which maintains a $\mathrm{C} 0$ continuous surface without bending, or adding stiffness to the structure. This was chosen as an optimal bound for a morphing skin that could maintain the airfoil shape without additional out-of-plane deformations due to aerodynamic loads and without additionally restricting actuation. The optimization of the skin covering this gap was considered outside of the realm of the current study; however it represents a continued challenge in morphing airfoil concepts. ${ }^{19}$

The SMA-driven hinge was modeled simply as a prescribed rotation about the discrete hinge because of the relatively high stiffness of the SMA wires compared to the Flexure Box. The Flexure Box was modeled in three 
parts. The unimorphs were modeled using a bending stiffness derived by the rule of mixtures, which was experimentally validated independently. The thick plastic pieces of the Flexure Box were modeled as rigid beams. The actual flexure mechanism, although composed of an elastomeric material was modeled with a linearly elastic material with an effective modulus that was determined by fitting experimental data for an applied tip load, shown in Figure 15. For this model, each elastomeric section in the flexure box and each unimorph were discretized into 20 evenly-spaced beam elements, resulting in 94 elements in the total modeling including rigid components.

To verify the validity of the code to capture non-elastomeric structures, the hinged-box mechanism developed by Bilgen et. al was modeled and experimentally validated in a similar manner ${ }^{8}$. The rigid hinges were modeled by decreasing the stiffness top-most and bottom-most elements of the flexure mechanism to near zero and increasing the stiffness in the other beams in the flexure mechanism to that equivalent of the rigid plastic.

As seen by the difference in smoothness between the flexure box and hinged box experimental data, the solidstate nature of the flexure box aids in eliminating frictional position errors. Additionally, the finite element results correlate well to the hinged box data. The flexure box modeled structure is generally slightly stiffer than the experimental results due to the elastomeric nature of the flexure box. However, it was assumed that the current model will adequately represent the structure when establishing trends about the effects of aerodynamic loading.

Using this model, the normalized actuation amounts $\left[\mathrm{MFC}_{\delta}, \mathrm{SMA}_{\delta}\right]$, were chosen so that the static, unloaded tip deflection range for the two actuators were equivalent to the values previously experimentally measured. It is important to note that, for the coupled simulations, $\mathrm{MFC}_{\delta}$ prescribes an applied moment rather than experimentally measured shape. This allows for the simulation of the aeroelastic effects on the Flexure Box.

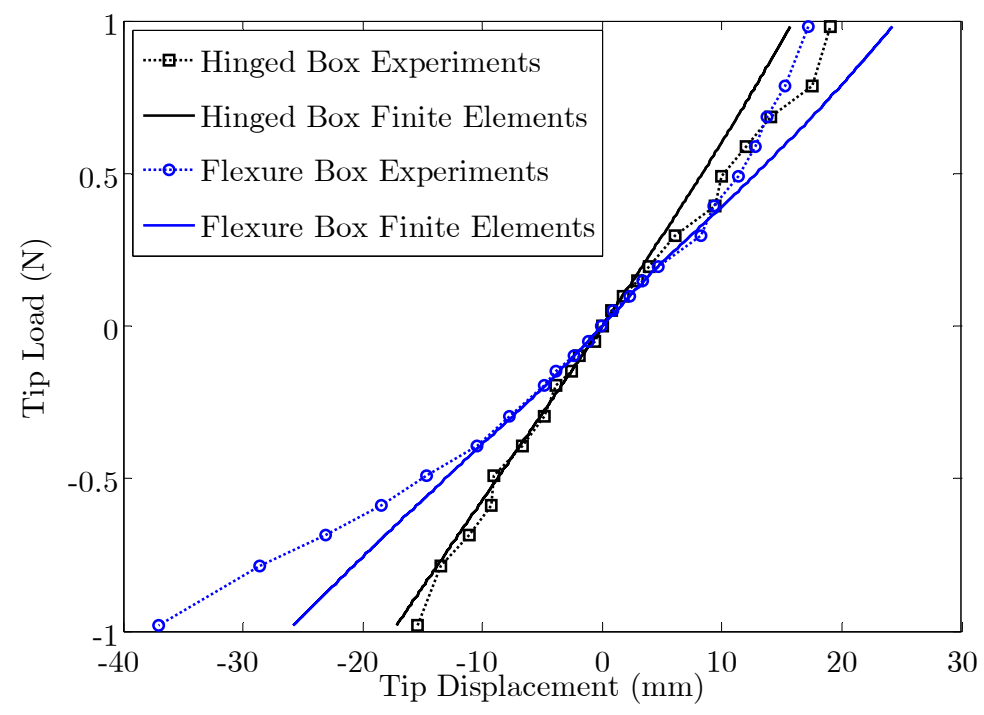

Figure 15: Experimental stiffness fitting of flexure box

\section{B. Coupling the structure and aerodynamics}

The corotational finite element code was coupled with the OVERTURNS flow solver by linearly interpolating the pressures on the surface to their respective beam elements at each iterative step. These pressures then followed their respective beam elements throughout the structural convergence step. The geometry for each converged structure was regenerated into a new grid for the CFD solution before each coupling, resulting in a loosely coupled convergence scheme. A flow chart diagraming the overall solution technique is shown in Figure 16. The various disciplines shown are as follows: structures in red, aerodynamics in blue, and coupling in green. Different from the iterative techniques conventionally used in corotational finite element codes was the added ability of the structural code to adapt the change in forces between load steps to prevent large changes in iterative updates for the geometry. This adaptive load step was important in ensuring convergence of the solution in early load steps and higher flow speeds where large changes in pressure between pressure updates could potentially cause the iterative solution to diverge. 


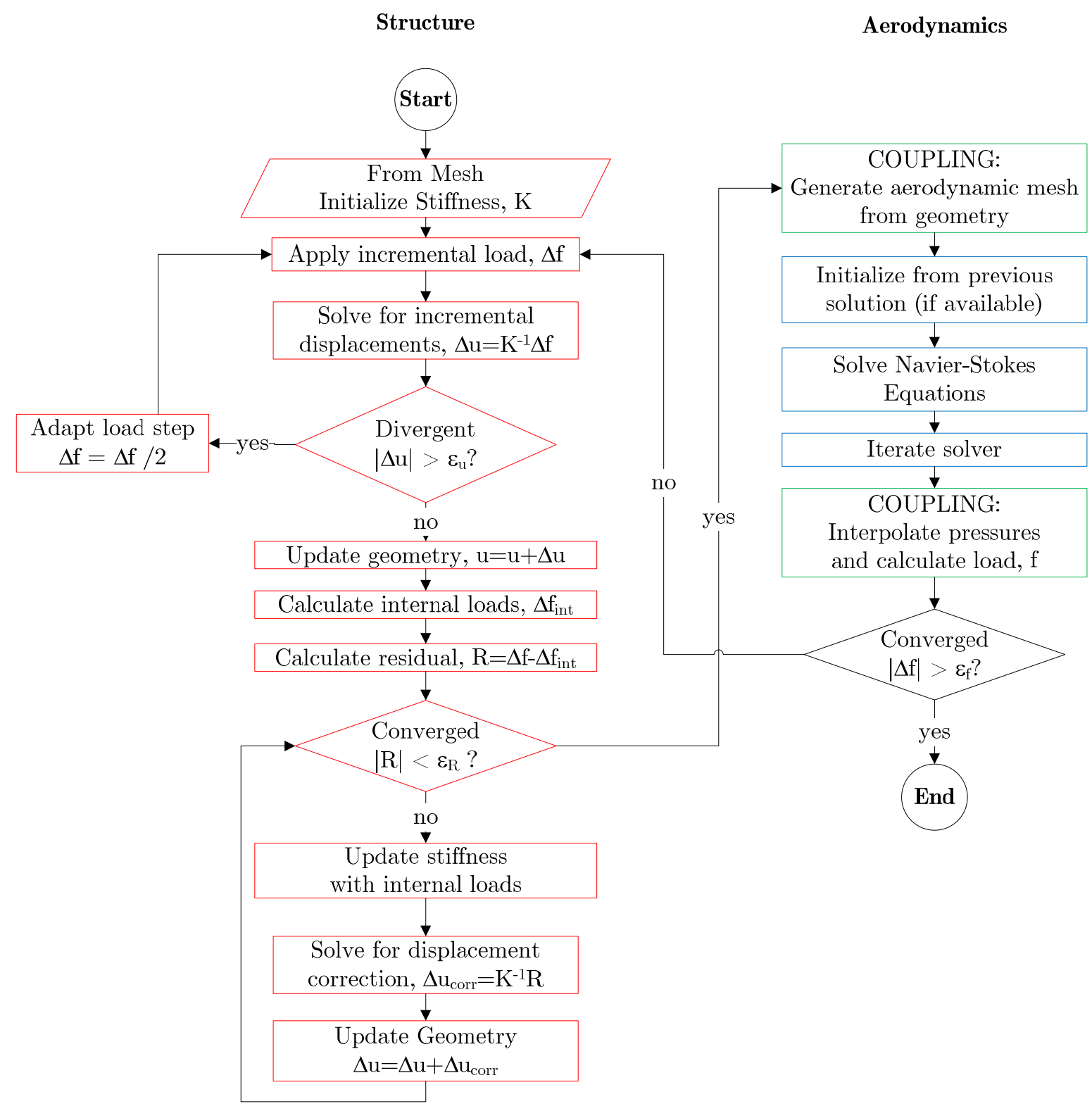

Figure 16: Flowchart of Structural / Aerodynamic Solution Coupling

\section{Coupled aero-structural optimization results}

Previously, the uncoupled results assumed that flow speed could only affect the actuation results through changing the Reynolds Number. By coupling the aerodynamic and structural solution, the aerodynamic loads also influenced the achievable configurations at each flow speed. The coupled finite element code detailed in previous sections was used to generate aerodynamic force coefficients over a range of flow speeds (10 to $30 \mathrm{~meter} / \mathrm{second}$ ) and angles of attack $\left(-15^{\circ}\right.$ to $\left.15^{\circ}\right)$. Using the same objective function and constraints as summarized in the uncoupled aerodynamic optimization Section II.D, the effect of flow speeds on the SMA, MFC and SSMA actuation schemes were evaluated. The Flexure Box structure for the SMA-only case was modeled as rigid because no compliant structures would be utilized in such a case. The sampled points are shown in blue and the contours are generated through cubic spline interpolation in Figure 17. 

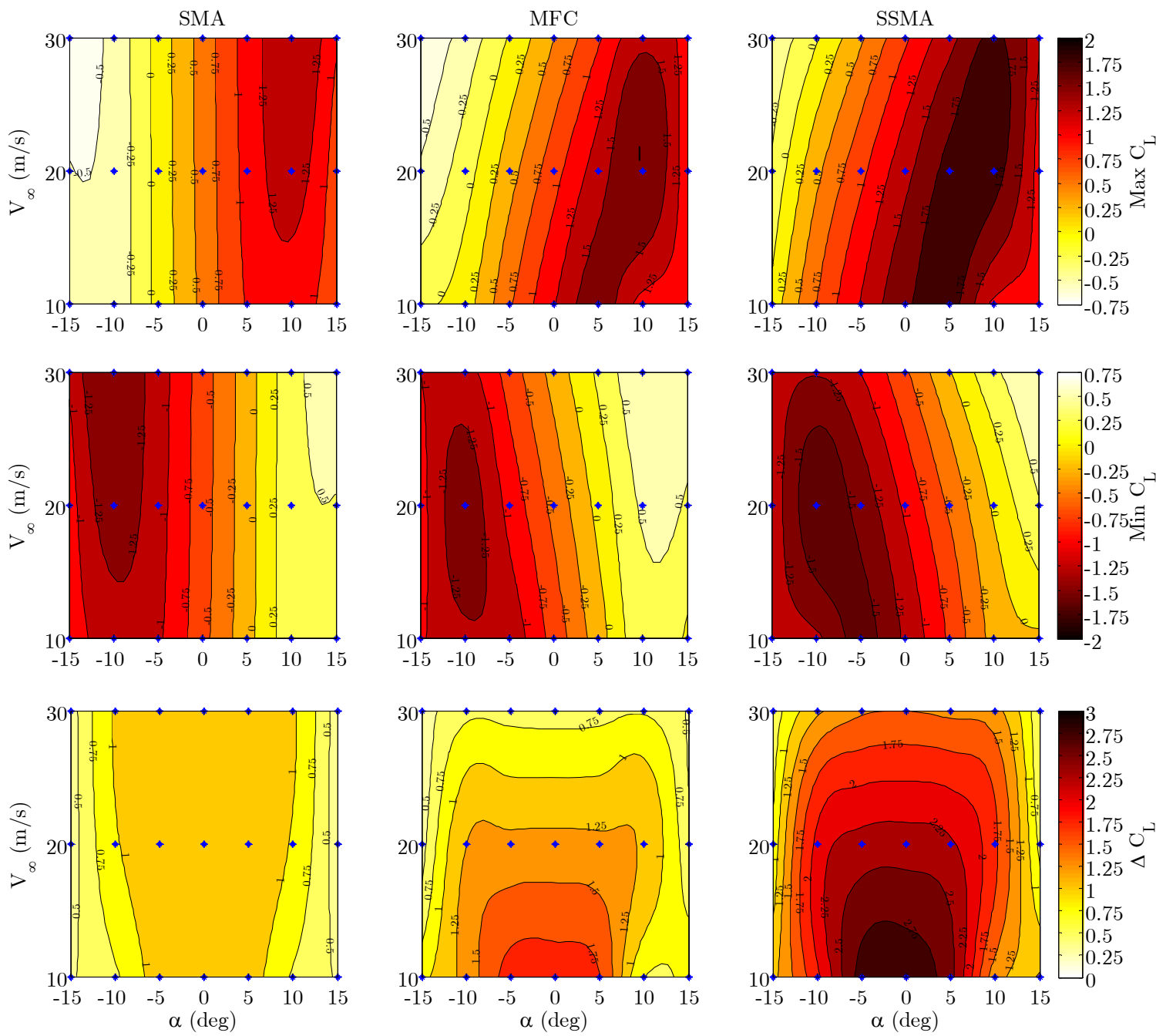

Figure 17: Coupled SMA, MFC, SSMA aerodynamic performance

(top row) max CL, (middle row) min CL, (bottom row) difference between max and min CL

In Figure 17, each row represents a different optimization and each column represents a different actuation scheme, allowing for direct comparison, between the SSMA and its constituent actuators. In each optimization, the ability of each actuator to influence the flow is emphasized. Accordingly, the desirable quantity is shown as dark while less desirable points are shown as lighter colors. Each row maintains the same color scheme for comparison of optimized quantities and each column represents a single actuator configuration.

Examining the left column, the SMA shows the effect of the discrete hinge with a rigid actuation mechanism on the flow. The maximum and minimum lift coefficient for the flow vary slightly due to Reynold's number effects. Thus the SMA actually becomes slightly more effective at higher flow speeds, especially near stall conditions and as seen before, the change in lift coefficient is symmetric about zero angle of attack.

In the middle column, the MFC shows a slightly more varied effect due to flow speeds, between $10-20 \mathrm{~m} / \mathrm{s}$ the actuator increases in effectiveness, achieving larger maximum and minimum lift coefficients, similar to the SMA. However, the ability of the actuator to affect the flow via $\Delta \mathrm{C}_{\mathrm{L}}$ decreases as flow speed increases. Until approximately $25 \mathrm{~m} / \mathrm{s}$ the MFC still exhibits superior actuation over the SMA, due to the conformal bending of the MFC to affect the flow despite reduced tip deflection.

In the right column, we see that the SSMA exhibits superior ability over the SMA and MFC at all tested flight conditions. Although the range is slightly asymmetric due to the MFC actuation effects, the two actuators work together to maximize the effect on the flow at all flight conditions, restoring most of the symmetry in $\Delta \mathrm{C}_{\mathrm{L}}$. Additionally, the SSMA experiences only $20 \%$ reduction in lift coefficient between $10-20 \mathrm{~m} / \mathrm{s}$. As previously stated, the modeled structure is slightly stiffer than experimental results. However, the aeroelastic simulations still reflect 
that the aerodynamic flow can effectively be influenced by the MFC despite aerodynamic reduction of the tip deflection.

\section{Reflex camber effect}

In addition to analyzing the ability of the concepts to influence the flow, it is important to analyze how they do so. Examining the effect of flow speed on maximum attainable lift coefficient for the SSMA, the optimization almost always finds that the optimal actuation configuration for maximum lift is the intuitive choice, actuating both the SMA and MFC $100 \%$ down, $\left[\mathrm{MFC}_{\delta}, \mathrm{SMA}_{\delta}\right]=[-1,-1]$. However, for high angles of attack, the optimizer finds a different result. Some points where this occurs are shown in Figure 18, indicated by points 1 through 4 .

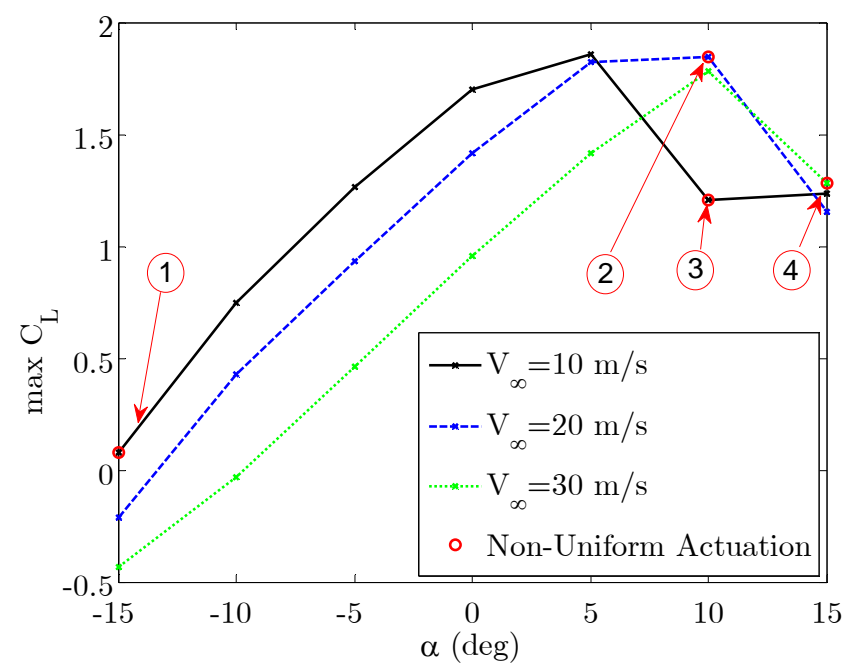

Figure 18: Sample of Non-Uniform Actuation Points

Point 3, which corresponds to $\mathrm{V}_{\infty}=10 \mathrm{~m} / \mathrm{s}$ and $\alpha=10^{\circ}$, shows an example of both SMA and MFC working together synergistically to effectively control the flow. For this low flow speed, high angle-of-attack flight condition, the "optimized" reflex actuation shows significantly less flow separation than the intuitive uniform actuation case, reflected by the attached streamlines in Figure 19. The optimizer incorrectly finished at a constrained local optimum which was less than the global optimum; however in the two cases the lift coefficients are nearly identical, seen in Table 2.This result is different from the uncoupled result in Section II.C., where the lift coefficient for the uniform actuation was much higher than the reflex actuation case. However, the drag and pitching moment for the reflex actuation case are still much lower. The pressure difference between the upper and lower surface near the tail is significantly less in the reflex configuration, also reflected in the decreased pitching moment.. Accordingly, less aerodynamic loading is placed on the Flexure Box in the reflex configuration.

Examining a higher loading case, point 4 , which corresponds to $V_{\infty}=30 \mathrm{~m} / \mathrm{s}$ and $\alpha=15^{\circ}$, reflex actuation is seen to further improve performance near stall for high flow speeds. In this case, the lift coefficient is dramatically improved in the reflex actuation case versus the uniform actuation case while still lowering the drag and pitching moment, as seen in Figure 20 and Table 2. This effect is largely due to aeroelastic deformations in the structure. In Figure 21 we see that the reflex actuation has prevented the flexure box from buckling, reflected in the decrease in curvature of the front flexure box between the uniform and reflex actuation configurations. Thus, the synergistic effects of reflex actuation are actually more effective as the aeroelastic effects on the airfoil grow and the SSMA concept can compensate for the lower stiffness of the Flexure Box. 

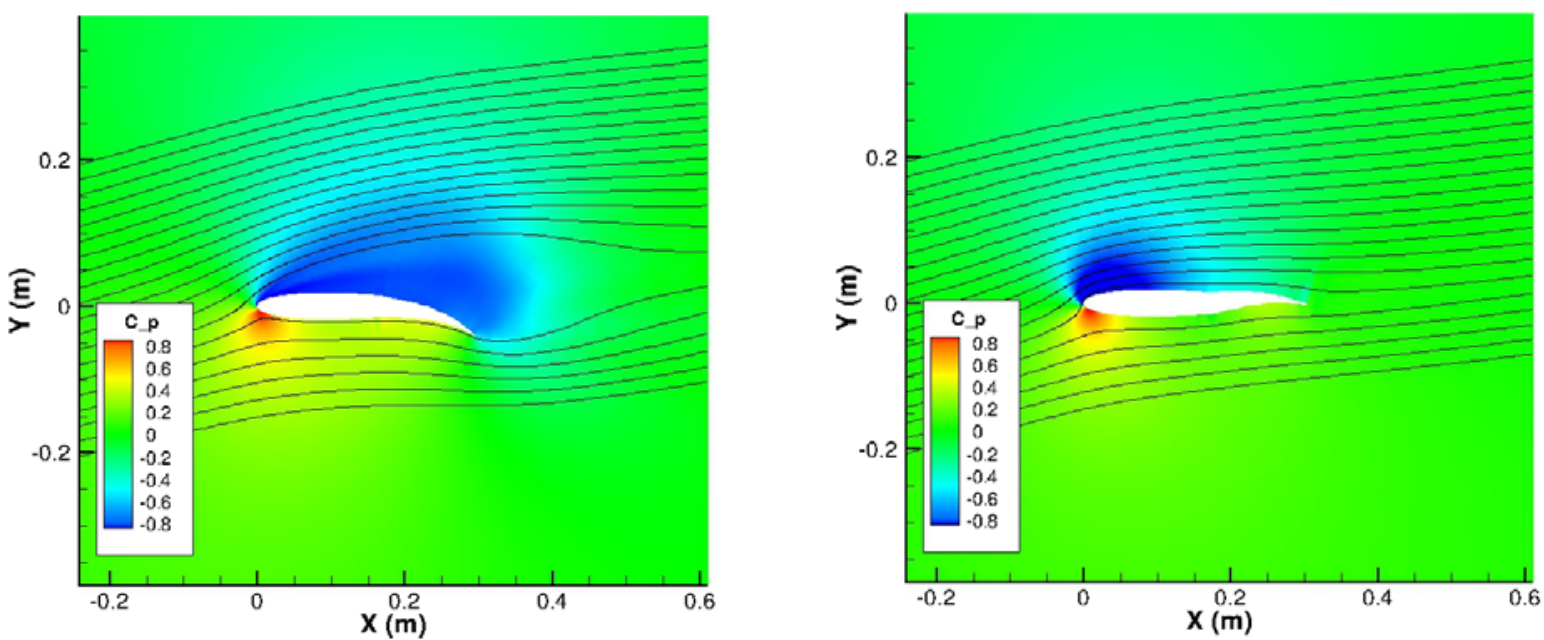

Figure 19: Optimizing SSMA for maximum Lift Coefficient $\left(C_{L}\right)$ for Point $\# 3\left(V_{\infty}=10 \mathrm{~m} / \mathrm{s}, \alpha=10^{\circ}\right)$ pressure coefficient for (left) Uniform Actuation (right) Reflex Actuation
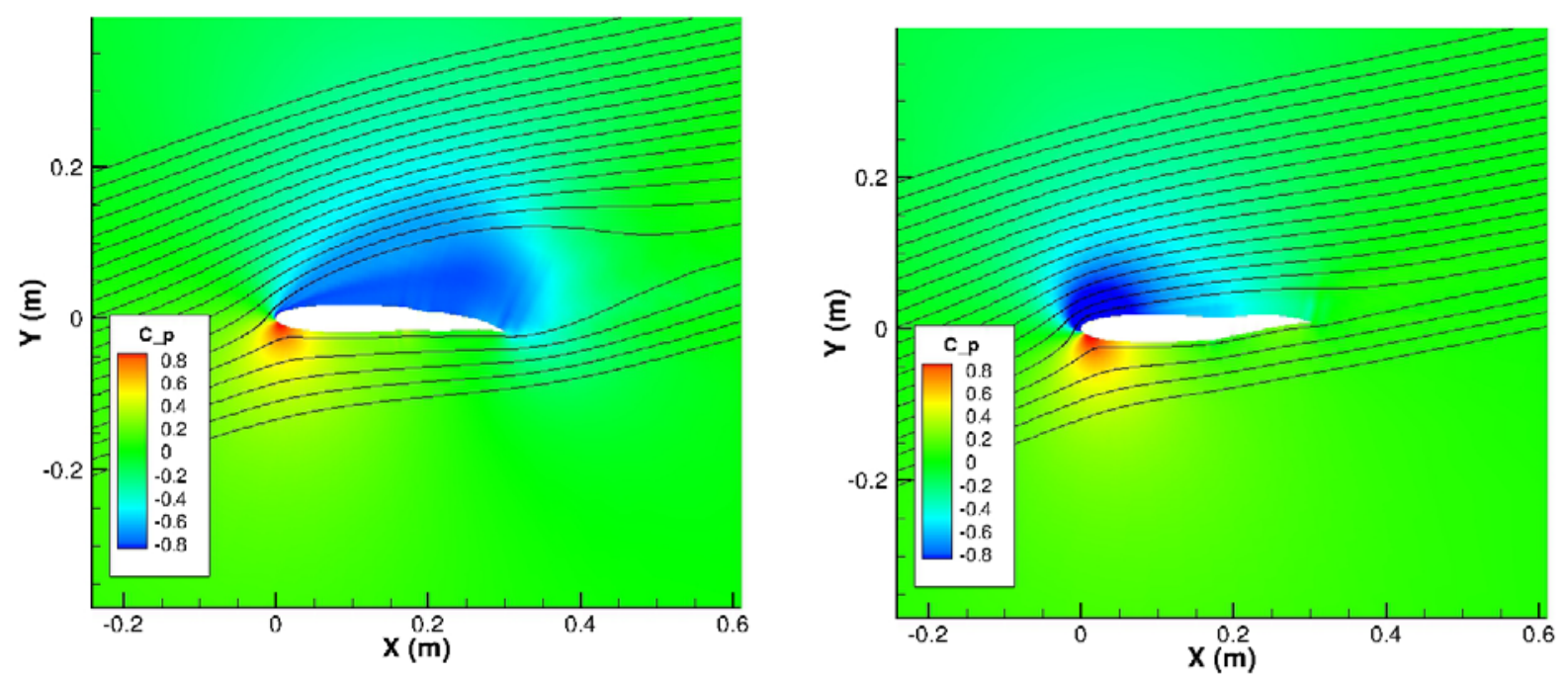

Figure 20: Optimizing SSMA for maximum Lift Coefficient $\left(C_{L}\right)$ for Point $\# 4\left(V_{\infty}=30 \mathrm{~m} / \mathrm{s}, \alpha=15^{\circ}\right)$ pressure coefficient for (left) Uniform Actuation (right) Reflex Actuation

Table 2: Sample of Reflex Actuation Effects

$$
\begin{aligned}
& \text { Point 3: } \quad \text { Point 4: } \\
& \left(V_{\infty}=10 \mathrm{~m} / \mathrm{s}, \alpha=10^{\circ}\right) \quad\left(V_{\infty}=30 \mathrm{~m} / \mathrm{s}, \alpha=15^{\circ}\right)
\end{aligned}
$$

$\begin{array}{ccccc}\text { Configuration } & \text { Uniform } & \text { Reflex } & \text { Uniform } & \text { Reflex } \\ \left.\mathbf{M F C}_{\boldsymbol{\delta}}, \mathbf{S M A}_{\boldsymbol{\delta}}\right] & {[-1,-1]} & {[-0.78,1]} & {[-1,-1]} & {[-0.86,1]} \\ \mathbf{C}_{\mathbf{l}} & 1.229 & 1.206 & 0.988 & 1.235 \\ \mathbf{C}_{\mathbf{d}} & 0.350 & 0.059 & 0.322 & 0.075 \\ \mathbf{C}_{\mathbf{m}} & -0.251 & -0.083 & -0.179 & -0.053\end{array}$

12

American Institute of Aeronautics and Astronautics 

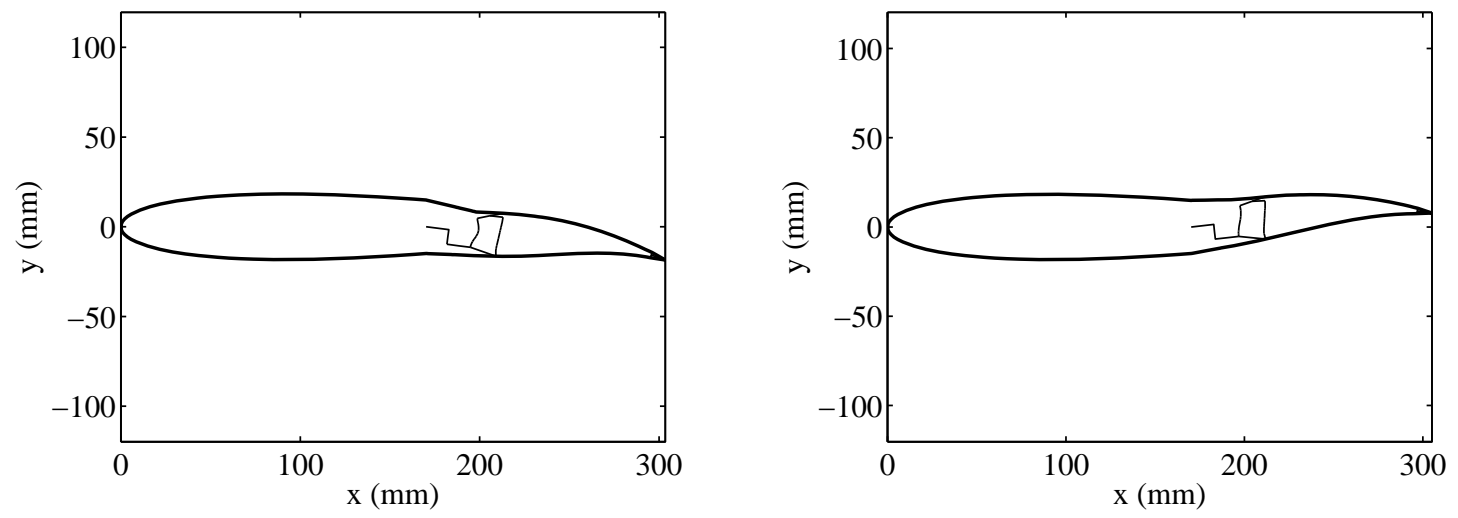

Figure 21: Structural Configurations for Point $\# 4\left(V_{\infty}=30 \mathrm{~m} / \mathrm{s}, \alpha=15^{\circ}\right)$ (left) Uniform Actuation

(right) Reflex Actuation

\section{Conclusions}

The results demonstrate that the SSMA concept can effectively utilize its constituent actuators (a discrete SMAdriven hinge, and a bending MFC-driven aileron) to achieve superior aerodynamic performance. These improvements were characterized using two methods: optimized static aerodynamic performance and optimized static aeroelastic performance.

The uncoupled aerodynamic performance was modeled using a Navier-Stokes flow simulation with experimentally-measured, unloaded airfoil shapes. Optimizing the performance of the MFC, SMA, and SSMA concepts over a range of flight conditions, the SSMA showed roughly $45 \%$ improvement over the MFC and $180 \%$ improvement over the SMA in influence over maximum change in airfoil lift coefficient at 20 meters/second.

Coupled aerodynamic performance was compared among the actuation concepts by coupling the Navier-Stokes solver with a geometrically nonlinear corotational finite element code. The structure of the actuators was modeled as Euler-Bernoulli beam elements while the actuation of the SMA and MFC were implemented as a discrete rotation and distributed applied moment, respectively. The coupled code simulated the effect of aerodynamic forces on the actuators and allowed for performance analysis under realistic loads. The optimized performance of the SSMA was compared to its constituent actuators and showed improved capability to influence the lift coefficient over flow speeds ranging from $10-30 \mathrm{~m} / \mathrm{s}$ and angles of attack between $-15^{\circ}$ and $15^{\circ}$. The SSMA also demonstrated superior flow control at flight conditions with high angles of attack by leveraging its unique reflex actuation capabilities to minimize flow separation and mitigate aeroelastic effects

Thus the SSMA concept was shown to create a truly synergistic flow actuator where the advantages of both the MFC (conformal bending) and SMA (resistance to aerodynamic loads) were effectively utilized. Additionally, the SSMA had an increased ability to influence flow, actuating both uniformly and through a novel reflex actuation scheme.

\section{Acknowledgments}

The authors would like to thank the University of Michigan and the Clarence L. "Kelly" Johnson collegiate professorship for the fellowship funding that made this research possible. The authors also acknowledge the Brazilian National Council for Scientific and Technological Development (CNPq) through the Brazilian National Institutes of Science and Technology (INCT-EIE) and the Group of Smart Materials and Systems (GMSINT) for their support. 


\section{References}

1 Weisshaar, T. A., "Morphing aircraft systems: Historical perspectives and future challenges," Journal of Aircraft, vol. 50, 2013, pp. 337-353.

2 Chinaud, M., Scheller, J., Rouchon, J., Duhayon, E., and Braza, M., "Hybrid electroactive wings morphing for aeronautic applications," Diffusion and Defect Data Pt.B: Solid State Phenomena, vol. 198, 2013, pp. 200-205.

3 Hines, L., Arabagi, V., and Sitti, M., "Shape memory polymer-based flexure stiffness control in a miniature flapping-wing robot," IEEE Transactions on Robotics, vol. 28, 2012, pp. 987-990.

4 Pankonien, A. M., Faria, C. T., and Inman, D. J., "Synergistic Smart Morphing Aileron," Proceedings of 54th AIAA/ASME/ASCE/AHS/ASC Structures, Structural Dynamics, and Materials Conference, 2013.

5 Wilkie, W. K., Bryant, R. G., High, J. W., Fox, R. L., Hellbaum, R. F., Jalink Jr., A., Little, B. D., and Mirick, P. H., "Lowcost piezocomposite actuator for structural control applications," Proceedings of SPIE - The International Society for Optical Engineering, vol. 3991, 2000, pp. 323-334.

6 Bilgen, O., Erturk, A., and Inman, D. J., "Analytical and experimental characterization of macro-fiber composite actuated thin clamped-free unimorph benders," Journal of Vibration and Acoustics, vol. 132, 2010, p. 051005.

7 Pankonien, A., and Inman, D. J., "Experimental testing of spanwise morphing trailing edge concept," Proceedings of SPIE The International Society for Optical Engineering, vol. 868815, 2013, pp. 1-13.

8 Bilgen, O., Kochersberger, K. B., Inman, D. J., and Ohanian III, O. J., "Novel, bidirectional, variable-camber airfoil via macro-fiber composite actuators," Journal of Aircraft, vol. 47, 2010, pp. 303-314.

9 Duraisamy, K., "Studies in tip vortex formation, evolution and control," PhD Dissertation, Dept of Aerospace Engineering, University of Maryland, 2005.

10 Lakshminarayan, V., "Computational Investigation of Micro-Scale Coaxial Rotor Aerodynamics in Hover," PhD Dissertation, Dept of Aerospace Engineering, University of Maryland, 2009.

11 Turkel, E., "Preconditioning techniques in computational fluid dynamics," Annual Review of Fluid Mechanics, vol. 31, 1999, pp. 385-416.

12 Spalart, P., and Allmaras, S., "A one-equation turbulence model for aerodynamic flows," 30th AIAA Aerospace Sciences Meeting and Exhibit, 1992.

13 Baeder, J., Duraisamy, K., and Lakshminarayan, V., "RANS Predictions of Complex Hovering Rotor Configurations: From Micro Scale to Full Scale," Computational Fluid Dynamics Journal, vol. 18, 2011, pp. 3-4.

14 Aranake, A. C., Lakshminarayan, V. K., and Duraisamy, K., "Assessment of transition model and CFD methodology for wind turbine flows," 30th AIAA Applied Aerodynamics Conference, 2012.

15 Nelder, J. A., and Mead, R., “A simplex method for function minimization,” The computer journal, vol. 7, 1965, pp. 308313.

16 Battini, J.-M., "Co-rotational beam elements in instability problems," PhD Dissertation, Dept of Mechanics, Royal Institute of Technology, Sweden, 2002.

17 Relvas, A., and Suleman, A., "Application of the corotational structural kinematics and Euler flow to two-dimensional nonlinear aeroelasticity," Computers and Structures, vol. 85, 2007, pp. 1372-1381.

18 Crisfield, M., Remmers, J., and Verhoosel, C., Nonlinear finite element analysis of solids and structures, John Wiley \& Sons, 2012.

19 Thill, C., Etches, J., Bond, I., Potter, K., and Weaver, P., "Morphing skins,” The Aeronautical Journal, vol. 112, 2008, pp. $117-139$. 